\title{
Resíduos de agrotóxicos no leite humano e seus impactos na saúde materno- infantil: resultados de estudos brasileiros
}

\author{
Vanessa Fracaro Menck $^{1}$, Kathleen Grace Cossella ${ }^{2}$ e Julicristie Machado de Oliveira ${ }^{3}$
}

Os resíduos de alguns agrotóxicos podem ser detectados muitos anos após a exposição devido a sua alta estabilidade e característica lipossolúvel. O leite humano é um bom indicador da exposição ambiental e materna em decorrência da sua representativa fração lipídica e consequente presença de diversos xenobióticos. O objetivo deste artigo foi sistematizar os estudos brasileiros que avaliaram os resíduos de agrotóxicos no leite humano, que estimaram a exposição de gestantes e lactantes aos agrotóxicos e sua relação com desenvolvimento de defeitos congênitos. Os estudos foram identificados utilizando-se as seguintes palavras-chave (e seus equivalentes em inglês): "agrotóxicos", "pesticidas", "leite materno", "leite humano", "gestante", "recém nascido" e "Brasil". Foram selecionados 21 estudos, e em todos foi detectada a presença de ao menos um resíduo de agrotóxico no leite humano. Mulheres que tiveram maior número de gestações e que amamentaram por período mais longo apresentaram menores quantidades de resíduos. Exposição ocupacional e dieta rica em alimentos de origem animal, assim como a menor renda e escolaridade dos pais, estão possivelmente associadas a maiores concentrações de resíduos. A exposição durante a gestação mostra-se mais nociva ao desenvolvimento fetal.

Palavras-chave: agrotóxicos, leite humano, amamentação, neonatos, defeitos congênitos.

\section{Effects of pesticides in maternal and child health and concentration of residue in human milk: results of Brazilian studies}

The residues of some pesticides can be detected many years after the exposure due to their high stability and lipo soluble characteristic. Human milk is a good indicator of the environmental and maternal exposure due to its representative lipid fraction and the consequent presence of various xenobiotics, such as pesticides. The objective of this study was tore view Brazilian studies that had evaluated residues of pesticides in human milk, measured exposure of pregnant women and nursing mothers to pesticides and their relationship with the development of birth defects. The studies were identified by using the following keywords in English: "pesticides", "breast milk", "human milk", "pregnant women", "newborn" and "Brazil". We have selected 21 studies and, in all cases, it has been detected the presence of, at least, one pesticide in the human milk. Mothers with a higher number of pregnancies and longer breast feeding presented smaller quantities of residues. The occupational exposure and a diet rich in foods of animal origin, as well as the lower income and educational level of parents, are possibly

${ }^{1}$ Graduada em Nutrição pela Faculdade de Ciências Aplicadas - FCA, Universidade Estadual de Campinas - UNICAMP. Correspondência: Rua Pedro Zaccaria no 1.300, Caixa Postal 1.068, CEP: 13484-350. Limeira, São Paulo. Telefone: (19)98872-0845. E-mail: vanessa.menck@gmail.com.

2 Graduada em Nutrição pela Faculdade de Ciências Aplicadas - FCA, Universidade Estadual de Campinas - UNICAMP.

${ }^{3}$ Professora Doutora. Faculdade de Ciências Aplicadas - FCA, Universidade Estadual de Campinas - UNICAMP. 
associated to higher concentrations of residues. The exposure during pregnancy seems to be more harmful to fetal development.

Key-words: pesticides, human milk, breastfeeding, newborns infants, birth defects.

\section{INTRODUÇÃO}

O Brasil é hoje o terceiro maior produtor e exportador de alimentos do mundo de acordo com a Organização Mundial do Comércio [1]. Em relação às commodities alimentares, é líder nas exportações de suco de laranja, café, açúcar e álcool, além de ser o segundo colocado em produção e exportação de soja e milho [2]. Dentre as commodities citadas, todas estão enquadradas em categorias que utilizam maiores quantidades de insumos químicos em sua produção, o que levou o Brasil a se tornar o maior consumidor de agrotóxicos do mundo $[3,4]$.

A exposição aos agrotóxicos, apesar de ser maior na população rural, não é necessariamente ocupacional. Na população urbana, a exposição se dá principalmente pelos alimentos e pelo ambiente (ar, água, solo) [5]. Existe uma grande diversidade de substâncias utilizadas na agricultura, sendo que a maioria delas é estável quimicamente e tem grande potencial de bioacumulação, o que significa que ao longo da cadeia alimentar sua concentração aumenta e não há degradação. Além disto, muitas substâncias são lipossolúveis, portanto passíveis de serem armazenadas no tecido adiposo $[6,7,8]$, como é o caso do agrotóxico organoclorado DDT (Diclorodifeniltricloroetano) e seus metabólitos [8,9].

O ser humano, por comer uma grande variedade de alimentos e se posicionar no topo da cadeia alimentar, está potencialmente exposto a uma gama de substâncias que outros animais considerados comestíveis ingeriram, além da contaminação ambiental [10]. Ademais, a presente epidemia de sobrepeso e obesidade, associada a alto consumo de alimentos ultraprocessados ricos em gordura [11], fomenta um olhar mais reflexivo e cauteloso em relação às incertezas e aos riscos de bioacumulação de substâncias no organismo humano. Assim, atenção especial deve ser direcionada ao grupo materno-infantil devido ao fato do leite humano, por ter alta concentração de gordura, ser um potencial veículo de excreção de resíduos de agrotóxicos lipossolúveis presentes no tecido adiposo das mulheres [8]. Durante o período de gestação e amamentação, grandes ações metabólicas ocorrem e os estoques provenientes do tecido adiposo materno são mobilizados para a produção de leite para amamentação [12].

Há ainda poucos estudos brasileiros que buscaram estabelecer uma relação entre a exposição dos pais aos agrotóxicos e os efeitos na gestação e na saúde infantil. O leite humano é um bom indicador de contaminação ambiental por agrotóxicos, pois reflete a exposição ao meio ambiente e a dieta materna $[6,13]$. Sendo assim, o objetivo deste estudo foi sistematizar e analisar os resultados de estudos que avaliaram a concentração de agrotóxicos no leite humano e seus impactos na saúde materno-infantil durante a gestação e lactação.

\section{Fontes de dados}

Para a presente revisão, foram selecionadas as seguintes bases de dados bibliográficos: LILACSs, Banco de Teses da Capes, SciELO e Pubmed. As palavras-chave utilizadas foram: "agrotóxicos", "pesticidas", "leite materno", "leite humano", "gestante", "recém nascido" e "Brasil" e seus equivalentes em inglês. As buscas foram realizadas em maio de 2015. Foram localizados 56 estudos publicados entre 1989 e 2014, sendo que 12 estavam duplicados. Após a exclusão das duplicidades, os estudos foram avaliados e selecionados de acordo com os seguintes critérios: 1. artigo ou tese original, 2. exposição ocupacional materna ou paterna, 3. avaliação de impacto em grupo materno-infantil do Brasil, 4. determinação da concentração de resíduos de agrotóxicos no leite humano, 5. defeitos congênitos.

\section{RESULTADOS}

Foram selecionados 26 estudos que foram classificados em dois grupos, dentre eles, sete dissertações de mestrado, uma tese de doutorado e 18 artigos originais publicados em revistas nacionais e internacionais. O primeiro grupo é composto por oito estudos que se referem a pesquisas que avaliaram a concentração de resíduos agrotóxicos em leite humano. 
Como pode ser observado na Tabela 1, foram encontradas associações entre o número de gestações, período de amamentação e quantidade de resíduos de agrotóxicos no leite humano. De forma geral, quanto maior o número de gestações e de tempo de amamentação, menores as concentrações de resíduos no leite.

O segundo grupo é composto por 11 estudos que se referem a pesquisas que avaliaram a exposição aos agrotóxicos durante a gestação e o desenvolvimento de defeitos congênitos. Como pode ser observada na Tabela 2, a exposição ocupacional materna e/ou paterna, assim como a menor renda e escolaridade, está associada aos nascimentos com defeitos congênitos. Não há concordância entre os estudos em relação aos desfechos entre a exposição aos agrotóxicos e baixo peso ao nascer e nem com a quantidades de resíduos de agrotóxicos no leite e a idade materna.

Tabela 1. Artigos referentes à concentração de agrotóxicos no leite humano.

\begin{tabular}{l} 
Características dos Estudos \\
\hline 1. Sant'Ana et al. (1989) ${ }^{[13]}$ \\
- Estudo transversal \\
- Objetivo: analisar resíduos de agrotóxicos organoclorados \\
em amostras de leite humano. \\
- $\mathrm{n}=42$, mães de zona rural e urbana, Botucatu, SP.
\end{tabular}

2. Matuo et al. (1992) ${ }^{[14]}$

- Estudo transversal

- Objetivo: analisar resíduos de agrotóxicos organoclorados em amostras de leite humano (colostro).

- $\mathrm{n}=30$, mulheres internadas para o parto em Hospital

Universitário, Ribeirão Preto, SP.

\section{Beretta \& Dick (1994) ${ }^{[15]}$}

- Estudo transversal

- Objetivo: analisar resíduos de agrotóxicos organoclorados e outros em amostras de leite humano.

- $\mathrm{n}=30$, mulheres que viviam em zona urbana por no mínimo 5 anos, Porto Alegre, RS.

\section{Dorea (1997) ${ }^{[16]}$}

- Estudo transversal.

- Objetivo: analisar resíduos de organoclorados (DDT) em amostras de leite humano e relacionar com as mudanças que ocorrem no tecido adiposo durante a gestação.

- $n=40$, mulheres que realizaram cesáreas e que amamentaram seus filhos, Brasília, DF.

\section{Oliveira (1998) ${ }^{[17]}$}

- Estudo transversal

- Objetivo: analisar resíduos de agrotóxicos organoclorados em amostras de leite humano (colostro).

- $n=32$, mulheres que realizaram o parto no hospital Geral e Universitário Júlio Müller, Cuiabá, MT.
- Os níveis de DDT foram maiores em amostras da zona urbana;

- Mães da zona rural consumiam mais legumes e verduras e menos carne;

- Não houve associação entre os níveis de DDT e idade das mães;

- Mães fumantes apresentavam maiores concentrações de DDT;

- Dieldrin encontrado apenas em amostras da área urbana;

- Os organoclorados (DDT e HCH) apresentaram níveis reduzidos no organismo de acordo com o número de filhos amamentados por cada mãe.

- O agrotóxico Lindano foi detectado em 32\% das amostras, Heptacloro em 65\%, o Dieldrin foi detectado em uma amostra em altos níveis, o Aldrin não foi detectado;

- O DDT e o seu metabólito DDE foram encontrados em todas as amostras;

- Em mulheres expostas ocupacionamente, a concentração foi maior $(0,008$ a 0,455 $\mathrm{mg} / \mathrm{kg}$ ) do que e em mulheres não expostas (0,002 a 0,072 mg/kg);

- Foi encontrado decréscimo nos teores quando comparados aos estudos de 10 anos antes realizados na mesma área.

- As concentrações de organoclorados encontradas nas amostras variaram de 0,3 a 5,3 $\%$ em $100 \mathrm{ml}$ de leite humano;

- Ao menos dois compostos foram encontrados em cada amostra (DDE e HCH foram os mais comuns);

- 73\% das amostras passaram do limite tolerável para leite humano: 1,25 ug/ml;

- As mulheres com maior número de filhos e que amamentaram o primeiro filho por mais tempo tiveram menores níveis de DDE e DDT no leite. As oito mães que estavam amamentando seu primeiro filho tiveram amostras com maior concentração;

- Os agrotóxicos Aldrin, Oxyclordano, Aroclor e Transnoacloro tiverem seus valores abaixo do limite de detecção.

- Os pesos pré, pós-gestacional e variação na porcentagem de gordura corporal não estavam associados às concentrações de DDT no tecido adiposo materno abdominal e no leite humano;

- O número de filhos estava associado com as concentrações de agrotóxicos $(p=0,012)$; - Correlação significativa entre DDT total no tecido adiposo e concentração de agrotóxicos no leite humano $(r=0,709 ; p<0,001)$.

- 100\% das amostras apresentaram contaminação pelos agrotóxicos DDE e Lindano; - Resíduos de DDT foram detectados em 96,9\% amostras;

- Os agrotóxicos Aldrin e Heptacloro estavam em 9,4\% e 12,5\% das amostras, respectivamente;

- A maior quantidade de DDE do que de DDT sugere que as mães foram expostas a mais de um ano, tempo para o DDT ser metabolizado em DDE no corpo;

- As moradoras de zona rural possuíram níveis mais elevados de agrotóxicos no leite $(p<0,01)$. 


\section{Mello et al. (1999) ${ }^{[18]}$}

- Estudo transversal com o objetivo de analisar resíduos de agrotóxicos organoclorados em amostras de leite humano e leite bovino.

- $n=7$, mulheres residentes na Cidade dos Meninos, Rio de Janeiro, RJ.

\section{Mesquita (2001) ${ }^{[19]}$}

- Estudo transversal

- Objetivo: analisar resíduos de agrotóxicos organoclorados e em amostras de leite humano e relacionar os hábitos de vida e variáveis reprodutivas.

- $n=100$, mulheres doadoras do Banco de Leite do Instituto Fernandes Figueira, IFF/FIOCRUZ, Rio de Janeiro, RJ.

\section{Azeredo (2008) ${ }^{[21]}$}

- Estudo transversal

- Objetivo: analisar resíduos de agrotóxicos organoclorados em amostras de leite humano e estimar o consumo dessas substâncias por meio de Questionário de Frequência Alimentar (QFA).

- $\mathrm{n}=69$, mulheres residentes em cidades ao entorno do Rio Madeira, AC.

\section{Palma (2011) ${ }^{[6]}$}

- Estudo transversal

- Objetivo: analisar resíduos de agrotóxicos organoclorados em amostras de leite humano.

- $n=62$, mulheres entre $3^{a}$ e $8^{a}$ semana pós-parto, Lucas do Rio Verde, MT

\section{Corcellas et al. (2012) ${ }^{[22}$}

\section{- Estudo transversal}

- Objetivo: analisar resíduos de agrotóxicos piretóides em amostras de leite humano.

- $\mathrm{n}=20$, mulheres da Amazônia e Ronônia, Brasil; $\mathrm{n}=27$; mulheres de Santiago, Colômbia; $\mathrm{n}=6$, mulheres de Barcelona, Espanha.
- Leite humano:

- Valores para $\mathrm{HCH}$ :

- $\quad$ Cidade dos Meninos (0,0025 a 0,0337 mg.kg-1de leite)

- $\quad$ Outras regiões (valor máximo de 0,0013 mg.kg-1de leite) - Valores para HCH/ DDE

- $\quad$ Cidade dos Meninos (0,0130 a 0,0178 mg.kg-1de leite; 0,0006 a 0,0059 mg.kg-1de leite)

- Outras regiões (valor máximo de 0,0079 mg.kg-1 de leite; 0,0005 a 0,0016 mg.kg-1de leite)

- A diferença foi estatisticamente significante $(p>0,05)$ para o metabólito $\mathrm{HCH}$;

- Todas as amostras de leite das mães expostas apresentaram resultados positivos para DDT (0,0019 a 0,0285 mg.kg-1);

- Sem relação entre quantidade, idade e concentração de agrotóxicos encontrada.

- 100\% das amostras continham DDE, $90 \%$ DDT e $84 \% \beta$ - $\mathrm{HCH}$

- $18 \%$ das doadoras estavam com níveis de agrotóxicos no leite humano acima dos recomendados para ingestão diária de acordo com o Codex Alimentarius ${ }^{[20]}$ para lactentes; - Não houve correlação significativa entre idade e concentração de resíduos $(r=0,145$; $p=0,315)$;

- Em relação ao número total de filhos e ao tempo de amamentação do primeiro bebê, observa-se uma correlação negativa com os níveis de DDE $(r=-0,187 ; p=0,192$ e $r=-$ $0,059 ; p=0,683)$;

- A IDA (ingestão diária aceitável) média calculada para o $\sum$ DDT foi de 0,00341 mg.kg1 de peso.dia-1, inferior ao valor de referência do Codex Alimentarius que é de 0,005 mg.kg-1 de peso.dia-1;

- Todas as amostras apresentaram contaminação multiresidual, sendo que a maioria estava abaixo do limiar estabelecido. As amostras de Duque de Caxias tiverem maiores níveis de $\mathrm{HCH}$, o que pode estar relacionado com contaminação ocorrida em acidente na década de 60;

- As mulheres com mais filhos e que amamentaram o primeiro filho por mais tempo tiveram tendência a menores níveis de DDE (resultante da degradação do DDT).

- 100\% das amostras com contaminação de DDT e seus metabólitos;

- O maior valor de DDT encontrado foi em uma mãe primípara;

- O menor número que filhos e maior idade foram considerados fatores determinantes para maiores concentrações de resíduos de agrotóxicos organoclorados;

- Foi estimado o consumo de resíduos de agrotóxicos organoclorados pelos neonatos de acordo com as quantidades encontradas no leite e estes dados foram comparados com o limite de $0,020 \mathrm{mg}$ de DDT por $\mathrm{kg}$ de peso proposto pela WHO. Os valores encontrados variaram entre 0,00023 - 0,8322. De acordo com as amostras, $8,7 \%$ das crianças apresentaram consumo maior do que o limite tolerável por dia;

- A dieta das mães era baseada principalmente em farinha de mandioca, frutas e peixes de acordo com o questionário de frequência alimentar aplicado;

- O leite e a carne não foram mencionados em $98 \%$ dos QFA;

- Nenhuma das mães teve contato com o agrotóxico DDT diretamente e nem foi exposta ocupacionalmente na agricultura ao agente químico;

- O consumo de peixe foi considerado a fonte mais importante de contaminação das mulheres pesquisadas.

- Em todas as amostras foram encontradas contaminação por algum agrotóxico, sendo o DDE o mais comum;

- DDE (0,32-12,03 $\mu \mathrm{g}$ g-1 de gordura), 44\% por Endossulfam $\left(0,54-0,61 \mu \mathrm{g} \mathrm{g}^{-1} \mathrm{de}\right.$ gordura) e $13 \%$ por DDT $\left(2,62-12,4 \mu g g^{-1}\right)$. Os outros agrotóxicos estavam abaixo dos limites de detecção;

- Já ter tido aborto esteve relacionado à presença de pelo menos três substâncias no leite pela análise de regressão.

- Em cada país, um tipo de piretóide foi mais predominante de acordo com o mais utilizado no combate a Malária;

- As concentrações encontradas variaram entre 1,45-24,2 $\mu \mathrm{g} \mathrm{g}^{-1}$;

- Quanto maior o número de gestações, menores as concentrações de resíduos de agrotóxicos no leite humano encontradas em todos os países;

- No Brasil, foram avaliadas regiões que utilizaram essas substâncias também no combate a dengue, nestas os valores maiores foram encontrados, principalmente onde o uso massivo for permitido, chegando a $128 \mu \mathrm{g} \mathrm{g}^{-1}$;

- Quando calculada a estimativa de ingestão diária, em todos os casos estiveram próximas às recomendações máximas da OMS 
Tabela 2. Artigos referentes à exposição de agrotóxicos de gestantes e lactantes e o desenvolvimento de defeitos congênitos.

\begin{tabular}{|c|c|}
\hline Características dos Estudos & Resultados \\
\hline $\begin{array}{l}\text { 1. Gaspari et al. (2012) }{ }^{[23]} \\
\text { - Estudo transversal } \\
\text { - Objetivo: investigas a incidência malformações genitais em } \\
\text { neonatos. } \\
\text { - } \mathrm{n}=2.710 \text {, neonatos internados em hospital cujos pais } \\
\text { tiveram contato direto com agrotóxicos, Campina Grande, } \\
\text { PB. }\end{array}$ & $\begin{array}{l}\text { - } 80,36 \% \text { das mães e } 58,93 \% \text { dos pais reportaram uso de agrotóxicos } \\
\text { ocupacionamente; } \\
\text { - Foram identificados } 56 \text { casos de malformações genitais }(2,07 \%) \text {, com produção } \\
\text { normal de testosterona em todos os casos; } \\
\text { - Não foram identificadas mutações genéticas e, em apenas } 3(0,11 \%) \text { casos, foi } \\
\text { detectado polimorfismo; } \\
\text { - Dos } 92.86 \% \text { pais que alegaram uso doméstico dos agrotóxicos, } 89,29 \% \text { afirmaram } \\
\text { ter sido durante a gestação ou próximo ao período de fertilização; } \\
\text { - Estudo sugere que a exposição dos pais e a contaminação fetal podem ser } \\
\text { considerados fatores de risco para micropênis (alta prevalência) e outras malformações } \\
\text { genitais. }\end{array}$ \\
\hline
\end{tabular}

\section{Gonçalves e Silva et al (2010) ${ }^{[24]}$}

- Estudo caso-controle

- Objetivo: investigar a associação entre a exposição dos genitores aos agrotóxicos e o nascimento com defeitos congênitos.

- $n=126$, neonatos filhos de famílias residentes, Vale do São Francisco, SP.

- Foram selecionados 42 casos e 84 controles com equivalência de nível socioeconômico;

- Na grande maioria dos casos, os pais possuíam ensino fundamental incompleto e as mães eram do lar;

- Neonatos com defeitos congênitos foram mais expostos a agrotóxicos durante a gestação (OR ajustada $=1,5 ; \mathrm{IC} 95 \%=0,7-3,2 ; p=0,24)$;

- Observou-se associação entre a ocorrência de defeitos congênitos e o fato dos pais que trabalharem na agricultura e morarem próximos à lavoura $(O R$ ajustada $=1,2$; IC $95 \%=0,3-4,2 ; p=0,67$;

- Maior risco de desenvolver defeitos congênitos quando pelo menos um dos genitores foi exposto aos agrotóxicos (OR ajustada=1,3; IC $95 \%=0,4-3,9)$.

3. Siqueira et al. (2010) ${ }^{[25]}$

- Estudo ecológico.

- Objetivo: investigar a associação entre o uso de agrotóxicos, baixo peso ao nascer, anormalidades congênitas e morte fetal no Brasil.

$-\mathrm{n}=3.161 .176$, registros do ano de 2001 provenientes do sistema nacional de informações.

\section{Cremonese et al.(2012) ${ }^{[26]}$}

- Estudo ecológico.

- Objetivo: investigar a associação entre o consumo per capita de agrotóxicos e possíveis efeitos adversos na gravidez.

- $n=2.243 .514$, nascidos vivos do Paraná, Santa Catarina e Rio Grande do Sul, BR.

\section{Ferreira (2010) ${ }^{[27]}$}

- Estudo de caso-controle

- Objetivos: investigar a associação entre a exposição materna a fatores ambientais, aos agrotóxicos e o desenvolvimento de leucemia seus filhos.

- $\mathrm{n}=833$, lactentes na população brasileira no período de 1991 a 2007.

- Foram identificados 3.115.474 (nascidos vivos), 6.943 (mortes em menores de 1ano) e 38.759 (mortes fetais);

- Uso de agrotóxicos teve associação positiva com a mortalidade por anormalidades congênitas em menores de um ano $(p=0,001)$, e em maiores de 1 ano $(p=0,012)$;

- Uso de agrotóxicos teve associação positiva com anormalidade congênita $(p=0,022)$, e pré-maturos $(p=0,028)$;

- Não houve correlação entre uso de agrotóxicos e morte fetal ( $p=0,827)$;

- Possíveis efeitos da exposição aos agrotóxicos em relação ao baixo peso ao nascer e a mortalidade infantil por defeitos congênitos no Brasil.

- Nas capitais e suas respectivas microrregiões mais populosas foi encontrado maior consumo per capita de agrotóxicos;

- Escolaridade $\leq 8$ anos de estudos $(79,3 \%)$;

- 98\% das mulheres primíparas;

- A média geral de gasto com o consumo de agrotóxicos entre as 92 microrregiões para o ano de 1996 foi de $\mathrm{R} \$ 33,96 /$ habitante;

- Quando divididos em quartis de consumo, o primeiro quartil apresentou uma média de $R \$ 7,00 /$ habitante de gastos com agrotóxicos por pessoa, o segundo, $R \$$ $20,15 /$ habitante, o terceiro, $\mathrm{R} \$ 37,50 /$ habitante e o quarto $\mathrm{R} \$ 71,15 /$ habitante;

- Não foi observada relação entre o baixo peso ao nascer e defeitos congênitos.

- Foram selecionados lactantes de 0-24 meses e crianças de 2-12 anos, sendo 292 casos com leucemia e 541 controles;

- 45,2\% das mães relataram uso de agrotóxicos no período de gestação;

- Exposição aos agrotóxicos pode estar relacionada ao aumento do risco de desenvolver leucemia até 24 meses (OR ajustada=2,30; IC95\% =1,58-3,35);

- Maior risco de desenvolver a doença quando a exposição ocorre no primeiro trimestre da gestação $(O R$ ajustada=1,99; IC $95 \%=1,30-3,05)$ e durante a lactação $(O R$ ajustada $=2,08$; IC95\%=1,33-3,27);

- Exposição materna aos agrotóxicos aumenta o risco de leucemia (2-3 vezes) em menores de 24 meses;

- Escolaridade e renda materna foram maiores nos casos do que nos controles. 


\section{Rudge (2010)}

- Estudo transversal

- Objetivo: analisar resíduos de agrotóxicos organoclorados em amostras de leite humano.

- $\mathrm{n}=160$, mulheres, Botucatu, Ribeirão Preto, Campinas, Santos e São Paulo, SP.

\section{Silva (2009) ${ }^{[29]}$}

- Estudo transversal

- Objetivo: investigar a associação entre o teor resíduo de agrotóxicos organoclorados no soro de mulheres com o consumo alimentar estimado por meio QFA e tempo de residência na região.

- $n=924$, mulheres residentes na Cidade dos Meninos, Rio de Janeiro, RJ.
- Foram coletadas amostras de 20 mulheres de cada região selecionada, entre 2007 e 2008, e que residiam a, no mínimo, um ano antes da gestação no local de estudo;

- Em Botucatu e Ribeirão Preto foram selecionadas mulheres apenas de áreas rurais com predomínio da cultura de cana-de-açúcar;

- Na cidade de São Paulo amostras foram coletadas no hospital da UNIFESP, Hospital

e Maternidade Leonor Mendes de Barros e Vila Nova Cachoeirinha;

- O maior uso de agrotóxicos foi relatado nas áreas urbanas de São Paulo, 84 mulheres (53\% da amostra) relatara uso doméstico;

- 70 mulheres $(44 \%)$ das mulheres relaram que no mínimo uma pessoa é fumante na casa;

- Em todas as amostras, foi encontrado algum tipo de agrotóxico;

- DDE foi encontrado em $100 \%$ das amostras;

- Nas áreas rurais as amostras de leite analisadas obtiveram maiores concentrações e maior quantidade de agrotóxicos.

- Foram encontradas maiores concentrações de resíduos em comparação a outros estudos nacionais e internacionais com populações não expostas aos agrotóxicos; - O HCB foi o contaminante com menor média (1,18 $\mu$ g.L-1), o DDE com maior média de concentrações $(23,11 \mu \mathrm{g} \cdot \mathrm{L}-1)$. Todos os contaminantes apresentaram uma frequência superior a $80 \%$ e o DDE apresentou maior frequência, $97,4 \%$;

- Nas mulheres, todos os contaminantes sofrem uma queda em seus níveis na faixa de 11-20 anos, com valores significativamente inferiores $(p<0,05)$. Nos homens a queda dos níveis dos contaminantes ocorre na faixa de 21-30 anos, e também apresentou valores significativamente inferiores $(p<0,05)$;

- O consumo de frutas e leite procedentes de fora da CM e o consumo de legumes, verduras, ovos, manteiga e frango de procedência local estão associados a níveis $\geq 20$ ng.ml-1 de $\beta$-HCH, p,p'-DDE e p,p'-DDD respectivamente;

- Com relação aos alimentos procedentes do local, houve associação entre a frequência do consumo e os níveis sanguíneos $\geq 20$ ng.ml-1 de OC. Para o HCH, ficou evidenciada associação com alto consumo de carne vermelha; $\beta$-HCH com alto consumo de leite, frango e verduras e p,p'-DDE com alto consumo de legumes e verduras.

- Os municípios foram divididos em quartis conforme o consumo de agrotóxicos, resultando em quatro estratos contendo 77 municípios cada um, sendo o quarto quartil o de maior consumo;

- Dos 10 municípios localizados nas regiões de maior consumo do mapa, três apresentaram declínio significativo da proporção de nascimentos masculinos $(p<0,05)$ : Icaraíma, Palmeira e Barra do Jacaré;

- Com exceção de três, todos os municípios selecionados apresentaram proporção de nascimento masculino inferior a $50 \%$ em 2004, valores considerados não usuais (normal: superior a $51 \%$ );

- Fortes evidências de que a redução de nascimentos de homens seja influenciada por exposições químicas ambientais e ocupacionais a desreguladores endócrinos provenientes de diferentes fontes de contaminação;

- Os municípios considerados como maiores consumidores de agrotóxicos apresentaram tendência estatisticamente significante de declínio na proporção de nascimentos masculinos à medida que aumentaram os valores de consumo de agrotóxicos.

- Foram selecionadas 44 mulheres da região agrícola (15 lavradoras e 22 donas de casa) e 46 mulheres residentes em cidades sem exposição ocupacional ( 29 donas de casa e 24 que trabalhavam fora);

- Média de idade das mães: 22,8 anos;

- 90,91\% dos neonatos apresentava um peso ao nascer igual ou maior que $2.500 \mathrm{~g}$;

- A proporção de nascimentos com baixo peso de mulheres expostas ocupacionalmente foi maior do que nas não expostas, sendo $6,82 \%$ e $17,64 \%$, respectivamente;

- O mesmo padrão de alteração foi encontrado para neonatos com peso elevado: $9,09 \%$ e $11,76 \%$, respectivamente;

- Índice de Apgar não teve diferenças estatísticas entre o grupo de mães expostas e não expostas;

- Aumento significativo do número de neonatos pequenos para a idade gestacional quando relacionadas às mulheres expostas e não expostas (de 6,82\% para 17,64\%);

- A exposição de mulheres a múltiplos agrotóxicos ao longo de vários anos parece influenciar a curva de crescimento intrauterino. 


\begin{tabular}{ll} 
Continuação & \\
\hline 10. Sarcinelli (2001) ${ }^{[32]}$ & - Foi encontrada contaminação multiresidual de organoclorados persistentes nas \\
- Estudo transversal & amostras de soro materno, leite humano e sangue do cordão umbilical; \\
- Objetivo: investigar a associação entre a contaminação & - DDE presente em $97 \%$ das amostras pesquisadas de soro, 100\% das amostras de \\
multiresidual em amostras de soro e leite humanos e os & leite humano; \\
fatores socioeconômicos, reprodutivos e alimentares. & - O consumo regular de peixe e menor número de filhos estão relacionados com \\
$\mathrm{n}=72$, gestantes residentes na cidade, Rio de Janeiro, RJ. & maiores quantidades de resíduos de agrotóxicos no soro e leite materno. Mães \\
& nulíparas apresentaram maiores valores no soro; \\
& - Nas lactentes, a renda familiar, maior redução de IMC durante a amamentação e alto \\
& consumo de laticínios também foram fatores que contribuíram para maiores \\
& quantidades de contaminantes. \\
\hline
\end{tabular}

\section{DISCUSSÃO}

Para a presente revisão, não houve seleção de estudos de acordo com o tipo de agrotóxico analisado, porém foi possível observar a predominância dos agrotóxicos organoclorados. Estes foram amplamente utilizações em ações de saúde pública, principalmente no combate a vetores transmissores de doenças [33] até a década de 1990, além de terem sido empregados indiscriminadamente na agricultura e pecuária [4]. Ademais, por terem elevada estabilidade e serem passíveis de detecção mesmo após um longo período da exposição, muitas pesquisas foram e são desenvolvidas com o objetivo de determinar esta classe de agrotóxicos no leite humano ${ }^{[13,34]}$.

Há um consenso entre os diferentes estudos no que tange à relação entre o número de gestações e tempo de amamentação, pois foi possível constatar que mães com maior número de filhos e que amamentaram por mais tempo apresentaram menores níveis de resíduos de agrotóxicos no leite do que mães primíparas e que amamentaram por menos tempo $[13,14,15,16,19,21,22$, 24, 29,31, 32]. Em contrapartida, a idade materna parece não ser um fator que influencia a concentração de resíduos de agrotóxicos no leite.

Em todos os estudos incluídos na presente revisão e na totalidade das amostras analisadas nestes estudos, encontrou-se algum tipo de contaminante no leite humano. O DDE é o principal metabólito do DDT, agrotóxico da classe dos organoclorados, e o mais persistente e, portanto, foi encontrado em maiores concentrações [32]. A contaminação multiresidual também foi observada em alguns artigos. No estudo transversal de Sarcinelli (2001) [32], a contaminação de múltiplos resíduos foi encontrada no soro das mães, no leite humano e no cordão umbilical.

Uma série de estudos foi realizada no estado do Rio de Janeiro, na cidade de Duque de Caxias, em região próxima a uma fábrica desativada de agrotóxicos organoclorados - cujos produtos não foram retirados do local e estavam armazenados de forma precária e com vazamentos - foram observados maiores níveis de contaminantes nos alimentos, solo e leite humano [18, 19, 22, 35, 36]. Por ser uma região com grande produção de alimentos, durante as entrevistas questionou-se sobre a origem e frequência do consumo de alguns alimentos. Observou-se associação entre presença de $\mathrm{HCH}$ no leite humano e consumo de carne vermelha, de leite de vaca, frango e verduras e entre o DDE e consumo de verduras e legumes ${ }^{[29]}$.

No estudo de Corcellas (2012) [22], que comparou os valores de agrotóxicos piretóides no leite humano de mulheres residentes no Brasil, Espanha e Colômbia, concentrações mais elevadas foram encontradas nas amostras de mães moradoras dos bairros onde esta substância foi utilizada no combate a malária e na agricultura.

De maneira geral, a exposição a agrotóxicos em ambiente rural é maior do que a exposição em ambiente urbano [32, 33]. Pesquisas que avaliaram a dieta de mães por questionários de frequência alimentar apontam que o alto consumo de carne bovina, peixe e frango ${ }^{[21,29,32]}$ está relacionado a maiores concentrações de resíduos de agrotóxicos no leite humano. A hipótese explicativa para estes resultados refere-se ao fato dos alimentos de origem animal serem mais ricos em gordura e estarem mais sujeitos a bioacumulação e biomagnificação. O mesmo foi encontrado em estudos realizados na Índia [37] e na China [38], onde o alto consumo de peixes esteve associado a maiores concentrações de organoclorados no leite humano.

$\begin{array}{lrr}\text { Mulheres que estiveram } & \text { expostas } \\ \text { ocupacionalmente apresentaram } & \text { maiores } \\ \text { concentrações de resíduos de agrotóxicos no leite, além } \\ \text { da associação com defeitos congênitos }{ }^{[27]} \text {. O hábito de } \\ \text { comer nas plantações também estava relacionado com }\end{array}$


maiores efeitos nocivos ao feto [17, 22], assim como a

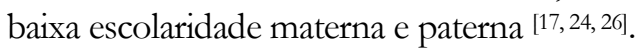

Gibson \& Koifman (2008) [30], em estudo ecológico que buscou conhecer a relação entre a utilização de agrotóxicos na agricultura e a distribuição de nascimentos no Paraná, observaram que os municípios com maior consumo apresentavam menor proporção de nascimentos masculinos. Em outro estudo que avaliou os impactos da exposição aos agrotóxicos no período gestacional, encontrou-se maior proporção de nascimentos femininos e, entre os masculinos, um maior risco de malformações genitais (micropênis, dentre outros). A hipótese é que isto ocorra em decorrência de alguns agrotóxicos serem desruptores endócrinos e terem efeitos estrogênicos e antiandrogênicos [23].

Resultados de outro estudo ecológico, que buscou conhecer a relação entre o uso de agrotóxicos e efeitos adversos nos desfechos gestacionais, apontam associação positiva com a mortalidade por anormalidades congênitas em menores de um ano [23]. Em relação ao efeito no peso ao nascer, em dois [25, 31] de três ${ }^{[25,31,26]}$ estudos, encontrou-se associação entre a exposição materna e menor peso.

Uma questão investigada em apenas um dos artigos revisados refere-se à possível relação entre a porcentagem de gordura corporal materna, o índice de massa corporal (IMC) com maior concentração de resíduos de agrotóxicos no leite humano. No estudo, porém, não foram encontradas associações entre estes fatores [29]. Resultados de pesquisas internacionais não são concordantes em relação a esta questão, pois há estudo que não relata associação [39] e há estudo que observou associação significante entre a concentração de resíduo de agrotóxico no leite humano e IMC materno [40].

No caso de mães fumantes, os níveis de organoclorados no soro e no leite foram maiores, pois o cigarro é uma fonte de contaminação para alguns agrotóxicos ${ }^{[22]}$. Por este motivo, em muitos estudos elas são retiradas da amostra.

Apenas um dos artigos problematiza o contraponto entre a contaminação do leite e os benefícios do aleitamento materno e reforça que a manutenção da prática é ainda a melhor alternativa para a manutenção da saúde do neonato. A Organização Mundial da Saúde (OMS) recomenda que todas as crianças sejam alimentadas exclusivamente com o leite humano até os seis meses de idade [41]. De forma alguma, a prática deve ser interrompida a não ser que haja alguma restrição séria por parte da mãe, pois o leite de vaca e outros alimentos também estão expostos aos mesmos contaminantes, podendo apresentar, inclusive, maiores quantidades ${ }^{[18]}$.

As diferenças de concentrações de resíduos de agrotóxicos organoclorados em diferentes estudos, no leite humano, podem ser atribuídas a fatores simultâneos tais como: idade, número de gestações, peso corpóreo, hábitos de dieta e período da coleta. Ademais, o momento da mamada e de realização da pesquisa, uma vez que a composição do leite tem intensas mudanças desde o colostro até o leite maduro, devem ser considerados, no entanto poucos estudos especificaram estas variáveis [6, 22].

Dependendo da categoria de agrotóxicos (composição química, estabilidade, solubilidade) seus efeitos são mais ou menos nocivos à saúde. Durante o período gestacional e de lactação, a vulnerabilidade é alta, pois os sistemas vitais e imunológico ainda estão em desenvolvimento. Além disso, não é possível prever quais os efeitos que a exposição nesta idade pode ocasionar em longo prazo e na idade adulta.

\section{CONCLUSÕES}

Mediante a análise dos artigos, evidencia-se a importância do controle na utilização de agrotóxicos, tanto na agricultura como nas ações em saúde, o que pode ser expandido também aos alimentos ultraprocessados e medicamentos de uso veterinário que estão expostos aos mesmos contaminantes. $\mathrm{O}$ grupo materno-infantil necessita de atenção especial em decorrência da importância do aleitamento materno no crescimento e desenvolvimento. $\mathrm{O}$ cuidado deve ser ainda maior nas áreas rurais, pois, além da exposição ser maior, o acesso aos serviços de saúde é mais restrito. A exposição aos agrotóxicos é incoerente com o conceito de segurança alimentar e nutricional e pode ser considerado um dos fatores relacionados à violação o direito humano a alimentação adequada, pois a amamentação é a melhor fonte alimentar do neonato.

\section{REFERÊNCIAS}

[1] Landim R. Brasil já é o terceiro maior exportador agrícola do mundo. Jornal O Estado de São Paulo [internet]. 06 mar 2010 [Acesso em 8 jun 2013]. Disponível em: http://www.estadao.com.br 
[2] Ministério da Agricultura, Pecuária e Abastecimento MAPA. Assessoria de Gestão Estratégica. 'Projeções do agronegócio de 2009/10 a 2019/2020. Brasília (DF); 2010.

[3] Sindicato Nacional das Indústrias de Defensivos Agrícolas (SINDAG). Dados de produção e consumo de agrotóxicos. Sindag News [internet]; 2013 [acesso em10 jun 2013]. Disponível em: http://sindag.org.br

[4] Associação Brasileira de Saúde Coletiva. Dossiê: Agrotóxicos, Segurança Alimentar e Saúde. 2012;1(1):15-16.

[5] Moreira P, Simões P, Dores V, Strussmann M. Contaminação de águas superficiais e de chuva por agrotóxicos em uma região de Mato Grosso. Ciênc Saúde Coletiva. 2012;17(6):1557-1568.

[6] Palma DCA. Agrotóxicos em leite humano de mães residentes em Lucas do Rio Verde - MT [dissertação]. Cuiabá: Faculdade de Saúde Coletiva da Universidade Federal de Mato Grosso; 2011.

[7] Meyer A, Sarcinelli PN, Moreira JC. Estarão alguns grupos populacionais brasileiros sujeitos à ação de disruptores endócrinos? Cad Saúde Pública. 1999;15(4):845-850.

[8] Brasil, Ministério da Saúde, Secretaria de Vigilância Sanitária, Departamento Técnico-Normativo, Divisão de Meio Ambiente e Ecologia Humana, Organização PanAmericana de Saúde. Manual de Vigilância da Saúde de Populações Expostas a Agrotóxicos. Brasília (DF); 1997.

[9] FAO/WHO. Evaluation of certain veterinary drugresidues in food. Thirty-fourth report of the Joint FAO/WHO Expert Committee on Food Additives. Geneva: World Health Organization; 1989. (WHO Technical Report Series; 788).

[10] D'Amato C, Torres JPM, Malm O. DDT (diclorodifeniltricloroetano): toxicidade e contaminação ambiental - uma revisão. Quím Nova. 2002;25(6a):995-1002.

[11] Monteiro C, Levy RB, Claro RM, Castro RR, Cannon G. Increasing consumption of ultra-processed foods and likely impact on human health: evidence from Brazil. Public Health Nutr. 2011;14(1):5-13.

[12] Dewey KG, Heinig MJ, Nommsen LA. Maternal weight loss patterns during prolonged lactation. Am J Clin Nutr. 1993;58:162-166.

[13] Sant'ana L, Vassilieff I, Lieselotte, J. Levels of organochlorineinsecticides in milk of mothers from urban and rural areas of Botucatu, SP, Brazil. B. Environ Contam Tox. 1989;42(6):911-918.
[14] Matuo Y, Lopes JN, Matuo T. Organochlorine pesticide residues in man milk in the Ribeirão Preto region, state of São Paulo, Brazil. Arch. Environ Contam Tox. 1992;22(2):167175.

[15] Bereta M, Dick T. Organochlorine compounds in human milk, Porto Alegre, Brazil. B. Environ Contam Tox. 1994;53(3):357-360.

[16] Dorea J, Granja A, Romero M. Pregnancy-related changes in fatmass and total DDT in breastmilk and maternal adipose tissue. Ann Nutr Metab. 1997:41(4):250-254.

[17] Oliveira M, Dores E. Níveis de praguicidas organoclorados no leite materno de uma população em Cuiabá - Mato Grosso. Rev Ecotoxicol Meio Ambiente. 1998;8:77-90.

[18] Mello JL. Avaliação da contaminação por HCH e DDT, dos leites de vaca e humano, provenientes da Cidade dos Meninos, Duque de Caxias - RJ [dissertação]. Rio de Janeiro: Escola Nacional de Saúde Pública/Fiocruz; 1998.

[19] Mesquita S. Avaliação da contaminação do leite materno por pesticidas organoclorados persistentes em mulheres doadoras do Banco de Leite do Instituto Fernandes Figueira, RJ [dissertação] Rio de Janeiro: Faculdade de Saúde Pública da Escola Nacional de Saúde Pública Sérgio Arouca; 2001.

[20] FAO/WHO. Codex Alimentarius. Pesticide Residues in food - maximum residue limits. 2a. ed. Geneva: World Health Organization; 2006.

[21] Corcellas C, Feo ML, Torres JP, Malm O, OcampoDuque W, Eljarrat E, et al. Pyrethroids in human breast milk: occurrence and nursing daily intake estimation. Environ Int. 2012;15(47):17-22.

[22] Azeredo A, Torres JPM, Fonseca MF, Britto JL, Bastos WR, Azevedo e Silva CE, et al. DDT and its metabolites in breast milk from the Madeira River basin in theAmazon, Brazil. Chemosphere. 2008;73(Supl 1):S246S251.

[23] Gaspari L, Sampaio DR, Paris F, Audran F, Orsini M, Neto JB, Sultan C. High prevalence of micropenis in 2.710 male new borns from an intensive use pesticide area of North eastern Brazil. Int J Androl. 2012;35(3):253-264.

[24] Gonçalves e Silva SR, Martins JL, Seixas S, Gomes da Silva DC, Lemos, SPP, Lemos, PVB. Defeitos congênitos e exposição a agrotóxicos no Vale do São Francisco. Rev Bras Ginecol Obstet. 2010;33(1):20-26. 
[25] Siqueira MT, Braga C, Cabral-Filho JE, Augusto LG, Figueiroa JN, Souza AI. Correlation between pesticide use in agriculture and adverse birth outcomes in Brazil: anecological study. B Environ Contam Toxicol. 2010;84(6):647-651.

[26] Cremonese C, Freire C, Meyer A, Koifman A. Exposição a agrotóxicos e eventos adversos na gravidez no Sul do Brasil, 1996-2000. Cad Saúde Pública. 2012;28(7):1263-1272.

[27] Ferreira J. Exposição pré-concepcional, gestacional e durante a lactação a pesticidas domésticos e outros contaminantes ambientais e leucemias em lactentes [dissertação]. Rio de Janeiro: Faculdade de Saúde Pública da Escola Nacional de Saúde Pública Sérgio Arouca; 2010.

[28] Rudge C. Níveis de substâncias tóxicas persistentes (PTS) em sangue de parturientes de sete áreas selecionadas do Estado de São Paulo - Brasil [dissertação]. Botucatu: Faculdade de Medicina da Universidade Júlio de Mesquita Filho; 2010.

[29] Silva G. Níveis de Agrotóxicos Organoclorados e Perfil Alimentar na Cidade dos Meninos Duque de Caxias, RJ, Brasil, entre 2003 e 2004 [dissertação]. Rio de Janeiro: Faculdade de Ciências da Saúde Pública e Meio Ambiente da Escola Nacional de Saúde Pública Sérgio Arouca; 2009.

[30] Gibson G, Koifman S. Consumo de agrotóxicos e distribuição temporal da proporção de nascimentos masculinos no Estado do Paraná, Brasil. Rev Panam Salud Publica. 2008;24(4):240-247.

[31] Freire F. Avaliação dos possíveis efeitos sobre o desfecho da gravidez em uma população de mulheres expostas cronicamente a agrotóxicos, na região do Vale de São Lourenço, Nova Friburgo, RJ [dissertação]. Rio de Janeiro: Faculdade de Saúde Pública da Escola Nacional de Saúde Pública Sérgio Arouca; 2005.

[32] Sarcinelli PN. Estudo dos níveis de pesticidas organoclorados persistentes em mulheres grávidas e lactantes no Rio de Janeiro [tese]. Rio de Janeiro: Faculdade de Biologia Celular e Molecular da Fundação Oswaldo Cruz; 2001.

[33] Sant'ana L, Vassilieff I, Lieselotte, J. Levels of organochlorine insecticides in milk of mothers from urban and rural areas of Botucatu, SP, Brazil. B Environ Contam Toxicol. 1989;42(6):911-918.

[34] Brasil. Ministério da Saúde. Agência Nacional de Vigilância Sanitária. Portaria no 10/85 - SNVS - MS, de 8 de março de 1985. Atribui à DINAL a elaboração da relação de substâncias com ação tóxica sobre animais ou plantas, cujo registro pode ser autorizado no Brasil, em atividades agropecuárias e em produtos domissanitários. Aprova as monografias técnicas e as classificações toxicológicas das formulações mistas. Atualizada até 30.06.96. Diário Oficial da União. 14 mar 1985.

[35] Oliveira RM. Estudo da contaminação do solo e pasto causada por hexaclorociclohexano $(\mathrm{HCH})$ na Cidade dos Meninos em Duque de Caxias, RJ [dissertação]. Rio de Janeiro: Escola Nacional de Saúde Pública; 1994.

[36] Braga AMCB. Contaminação ambiental por hexaclorociclohexano: estudo da exposição de escolares na Cidade dos Meninos, Duque de Caxias, Rio de Janeiro [dissertação]. Rio de Janeiro: Escola Nacional de Saúde Pública; 1996.

[37] Mannetje A, Coakley J, Bridgen P, Brooks C, Harrad $\mathrm{S}$, Smith $\mathrm{AH}$, et al. Current concentrations, temporal trends and determinants of persistent organic pollutants in breastmilk of New Zealandwomen. Sci Total Environ. 2013;458-460:399-407.

[38] Lu D, Wang D, Ni R, Lin Y, Feng C, Xu Q, et al. Organochlorine pesticides and their metabolites in human breast milk from Shanghai, China. Environ Sci Pollut Res Int. 2015;22(12).

[39] Song S, Ma X, Tong L, Tian Q, Huang Y, Yin S, Sun H. Residue levels of hexa chlorocyclohexane and dichlorodiphenyltrichloroethane in human milk collected from Beijing. Environ Monit Assess. 2013;185(9):72257229.

[40] Lee S, Kim S, Lee HK, Lee IS, Park J, Kim HJ, et al. Contamination of polychlorinated biphenyls and organochlorine pesticides in breast milk in Korea: timecourse variation, influencing factors, and exposure assessment. Chemosphere. 2013;93(8):1578-1585.

[41] Brasil, Ministério da Saúde, Secretaria de Política de Saúde, Organização Pan Americana da Saúde. Guia alimentar para crianças menores de dois anos. Brasilia (DF): Ministério da Saúde; 2002. 